\title{
sciendo
}

Transport and Telecommunication, 2021, volume 22, no. 2, 152-162

Transport and Telecommunication Institute, Lomonosova 1, Riga, LV-1019, Latvia DOI 10.2478/ttj-2021-0012

\section{HOW DOES PEDESTRIAN-DRIVER BEHAVIOR INFLUENCE IN THE NUMBER OF CRASHES? A MICHIGAN'S CASE STUDY}

\author{
Fadi Alhomaidat ${ }^{1}$, Lusanni Acosta-Rodriguez, ${ }^{2}$ \\ ${ }^{I}$ Department of Civil Engineering, Al-Hussein Bin Talal University \\ P.O. Box 20, Ma'an, Jordan \\ fadi.a.alhmedat@ahu.edu.jo \\ ${ }^{2}$ Department of Civil and Construction Engineering, Western Michigan University \\ 1903 W. Michigan Ave, Kalamazoo, Michigan, 49008 \\ lusanni.m.acostarodriguez@wmich.edu
}

\begin{abstract}
This study provides with a safety assessment of the pedestrian's crash data in one of the largest cities of the state of Michigan, Grand Rapids. Crash data reviewed included a 9-year period between years 2010 and 2018. Crash clusters with largest number of accidents were selected to perform analysis based on the normalization of crash with population (using Census Bureau information). Geographic Information System (GIS) software was used to gather this data using a 250-feet buffer around the clusters. Also, GIS was used to identify the infrastructure design and locations nearby the studied area (e.g. schools and hospitals) to understand the crash environments. Observation of the associated factors with pedestrian crashes were studied at the location of interest. An analysis of all safety efforts was completed and a list of recommendations and possible implementation strategies (e.g. pedestrian countermeasures). Finally, it was found that four types of pedestrian crashes were most representative that crashes involved left-turning vehicle, crashes involved right-turn vehicle, crashed involved pedestrian in crosswalk and through traffic, and pedestrian were not cross at designated cross location
\end{abstract}

Keywords: Pedestrians, Crash Data, GIS, Countermeasures, Case Study

\section{Introduction}

There is a need to find alternative active transportation modes especially with global increases; walking mode is being used for commuting and making trips. In Michigan, walking and running continue to raise every year. Unfortunately, pedestrian is expose to more severe injuries when involved in a crash. Understanding the location of pedestrian crashes is very critical to identify and implement appropriate countermeasures.

Many studies have been carried out in order to identify the consequences of contribution factors on pedestrian crash occurrences and severities. Contribution factors include pedestrian characteristics (gender and age) especially the vulnerable such as elderly and school-aged (Ang et al., 2017; Kim, 2019; O'Donovan et al., 2018) and males were found more involved in pedestrian-crashes than females (Chen et al., 2019; Newnam et al., 2018; Tulu et al., 2015), traffic characteristics (traffic volume and travel speed), intersection geometric features, neighbourhood environmental conditions (land use, access to public, and area types), visibility, alcohol, traffic control, location of crash, lighting condition, type of collision, and other factors (Alhajyaseen et al., 2013; Du et al., 2020; Iryo-Asano et al., 2015; Kim et al., 2010; Lam et al., 2014; Prato et al., 2019; Rankavat and Tiwari, 2016; Wang et al., 2016; Yao et al., 2015).

Road network structures have been studied in different ways, there are two types are the most common way that macro and micro level. Macro-level is typically continuous road over a certain part of the city, county, or state. In other hand, micro-level is not continuous over a part of a city, its work with neighbourhood level (Marshall and Garrick, 2011).

Wang and Kockelman (2013) performed a study to investigate Zone-based pedestrian crashes associated with factors such as land use, network, and demographic attributes. The study used a threeyears pedestrian crash data occurred in Austin, Texas. Crashes were categorized into two severity groups: severe injury including fatal and incapacitating, and non-severe injury including minor injury and noinjury. ArcGIS was used to aggregate the crash data from 2007-2009. Thiessen polygons were generated around each census tracts used. Spatial joint was used to aggregate the data in order to investigate 
contributing factors. Demographic attributes included: sidewalk density, resident's accessibility, roadway traffic density per class, population, and employment density. The study recommended to increase walking facilities such as sidewalk and pedestrian path, providing with pedestrian signals, to warn pedestrian on the time left to cross the street, in order to reduce crash rates. Finally, everyone is a pedestrian and while humans use this mode of transportation, they become exposed/vulnerable to be part of crash. Thus, understanding/accessing the risks/safety related to the pedestrian-driver conflicts is of crucial matter to transportation agencies, especially to make informed decisions. This paper is a case study of one of the largest cities in the state of Michigan, USA, Grand Rapids.

According to the Michigan Traffic Crash Facts (MTCF), there were 20,341 pedestrian crashes in Michigan from 2010-2018. Out of these, 1009 happened in the city of Grand Rapids (GR). The United States Census Bureau of 2018 shows that the population of GR was 200,136. So, the pedestrian crash ratio for Grand Rapids is 1:198 (one per every 198 people) yearly. Crash ratios of other larger Michigan cities picked for comparison are provided in Table 1.

Table 1. Pedestrian Population-Crash Ratio of Largest Cities in Michigan

\begin{tabular}{|l|l|l|l|l|}
\hline City & Population (2018) & Crashes (2010-2018) & Ratio & Rank \\
\hline Detroit & 672,977 & 4243 & $1: 159$ & 1 \\
\hline Grand Rapids & 200,136 & 1009 & $1: 198$ & 2 \\
\hline Flint & 95,926 & 435 & $1: 221$ & 3 \\
\hline Ann Arbor & 121,429 & 510 & $1: 238$ & 4 \\
\hline Lansing & 117,931 & 421 & $1: 280$ & 5 \\
\hline
\end{tabular}

Though not all cities in Michigan are presented in Table 1, one can see that among those presented (i.e. larger cities), the city of Grand Rapids is the second largest. Also, in this small presentation of the pedestrian crash ratio (population versus total crashes), it is observed that Grand Rapids falls into the second place. Overall, this study describes a spatial pedestrian crash analysis to quantify potential patterns behind the crash occurrence. More specifically, the primary objective of this study was to assess grand rapid pedestrian's crash data. A safety assessment was performed in the city to address the potential locations where pedestrian crashes are observed the most. The remainder of this paper is structured as follows, review of previous pedestrian crash data and census data, find associated human and infrastructure factors related to the crashes, and analysis and recommendations based on collected data.

\section{Research Methodology}

\subsection{General Descriptive Statistics}

Pedestrian crash data for 2010-2018 were collected from the Officials Highway Safety Planning (OHSP) crash records. Where exposure data such as population and public service's location (schools, hospital, etc.) were collected from America Factfinder and Michigan Department of Technology, Management and Budget, respectively. In this project, the state-wide pedestrian crash data. In addition, geographic map files for all 83 counties and the roads in Michigan were obtained from State of Michigan open data website to effectively display the pedestrian crash rates.

A GIS is software designed to manage, manipulate, and display of spatially referenced data. The 10th version of the Geographic Information System (GIS) software was used to plot the pedestrian crashes. Pedestrians crash GIS-based system was developed for the entire state of Michigan using nineyear pedestrian crash data. Selected crash categories and exposure such as population for each county were also included, in order to identify the county that experienced the highest level of pedestrian crashes. Pedestrian crash rates were calculated using GIS by incorporating color-coded maps with the entire roadway network for the state of Michigan.

Out of 1009 Pedestrian Crashes, 971 had coordinates to plot (96.23\% of useful data). So, Descriptive Statistics are based on those 971 Crashes. Figure 1 presents a broader information on pedestrian crashes per population in Michigan at a county level. In this density map, one can see that Kent county has a large number of pedestrian crashes when compared to the population in the area. The map is just for visualization purposes. Moreover, Figure 2 presents the distribution of pedestrian crashes in 
Grand rapids per year and severity. The figure also provides additional information on the injury severity of the pedestrian using the following codes: Square (fatal), triangle (injury), and circle (non-injury). Crash data included nine-year period crash data (2010-2018) where each year given different colour to distinguish these years. The map presented in Figure 2 helped us to identify areas where most of pedestrian crashes were occurring. This is expanded further in the paper.

\section{Total Pedestrian Crashes per Population (2010-2018)}

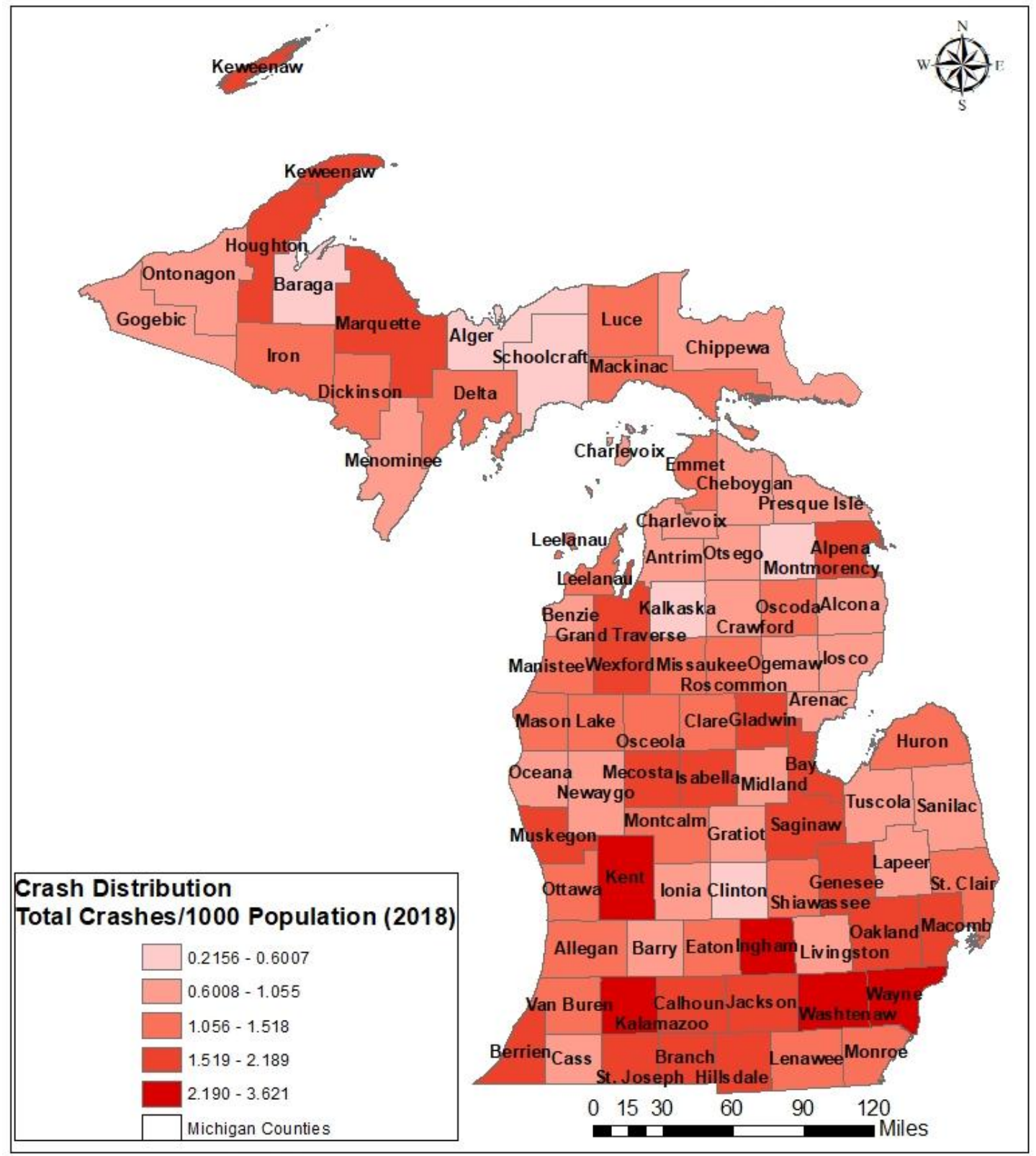

Figure 1. Pedestrian Crash per Population

Besides understanding and observing the locations where pedestrian crashes occurred in Grand Rapids, we also looked at other aspects of the crashes. Figure 3 presents pedestrian crashes per severity level and presents a summary information on the type of injury. From Figure 3, around fifty percent of the crashes are possible injured or level $\mathrm{C}$ (crash data dictionary terms for the least injury). The least frequent type is fatal crashes followed by non-injury crashes. Another relevant statistic is the location where the crash occurred classified by the Michigan Department of Transportation (MDOT). The distribution of the 
frequency of pedestrian crashes per area was also investigated. Most of the crashes occurred at intersections.

Another relevant descriptive statistic is the one related to alcohol involvement. The data showed that approximately $16 \%$ of the crashes were alcohol related. There were 395 people involved in alcohol related crashes: 184 drivers, 36 passengers and 175 pedestrians. Thus $46.58 \%$ of these were drivers, and $44.30 \%$ of them pedestrians $(9.11 \%$ were injured/uninjured passengers). Additional significant information to observe when dealing with crashes is the time of the day the crash occurred. This data is helpful as it depicts information on the environment when the pedestrian crashes occurred. In this case, the time of the day of the pedestrian crashes occurred the most in Grand Rapids. Most of the crashes occurred during lighted conditions: daylight and dark-lighted. Dark-lighted refers to the lighting coming from the streetlamps at night. It is expected to find more pedestrians during daytime than in any other time of the day.

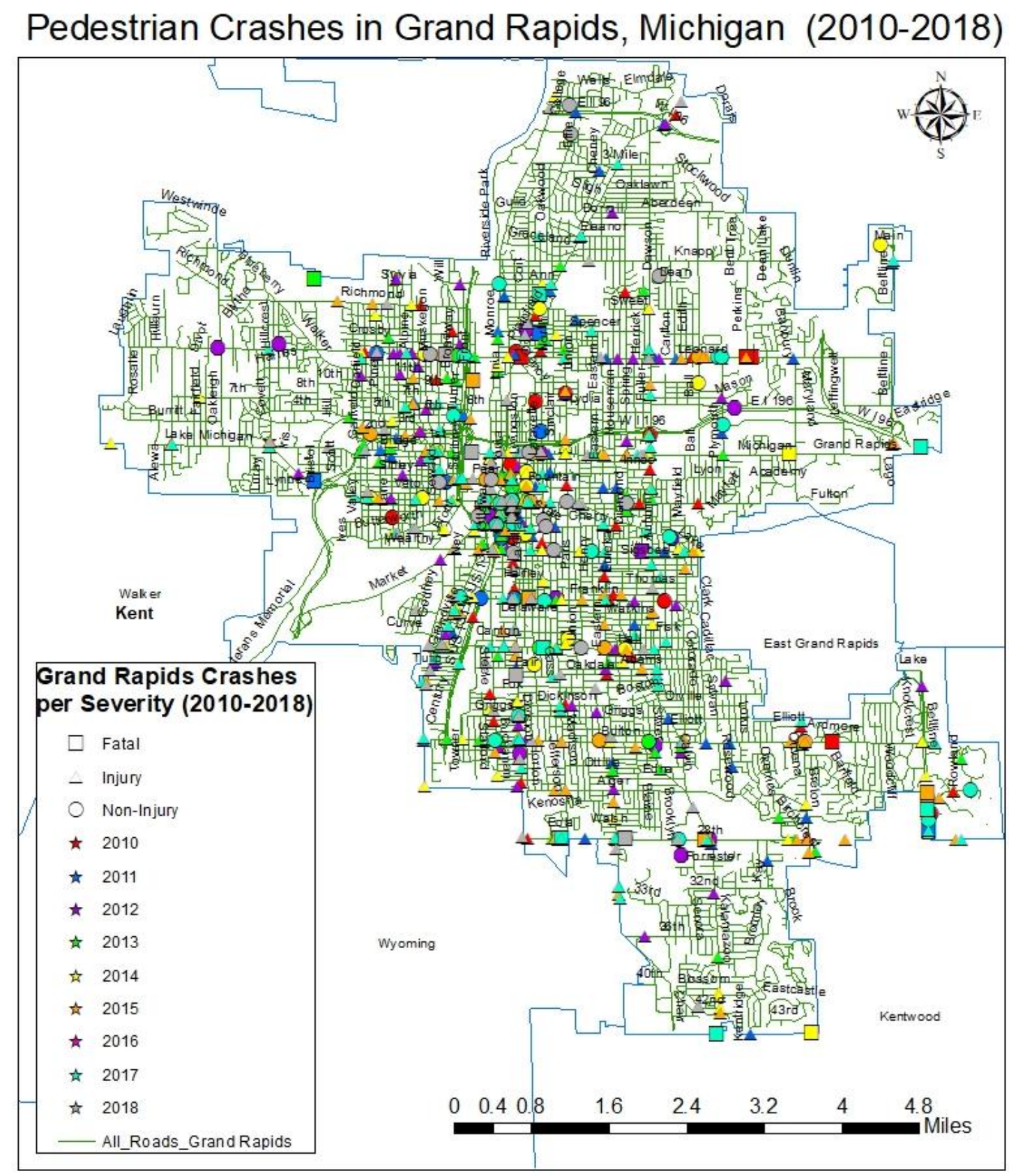

Figure 2.Pedestrian crashes per Severity in Grand Rapids 


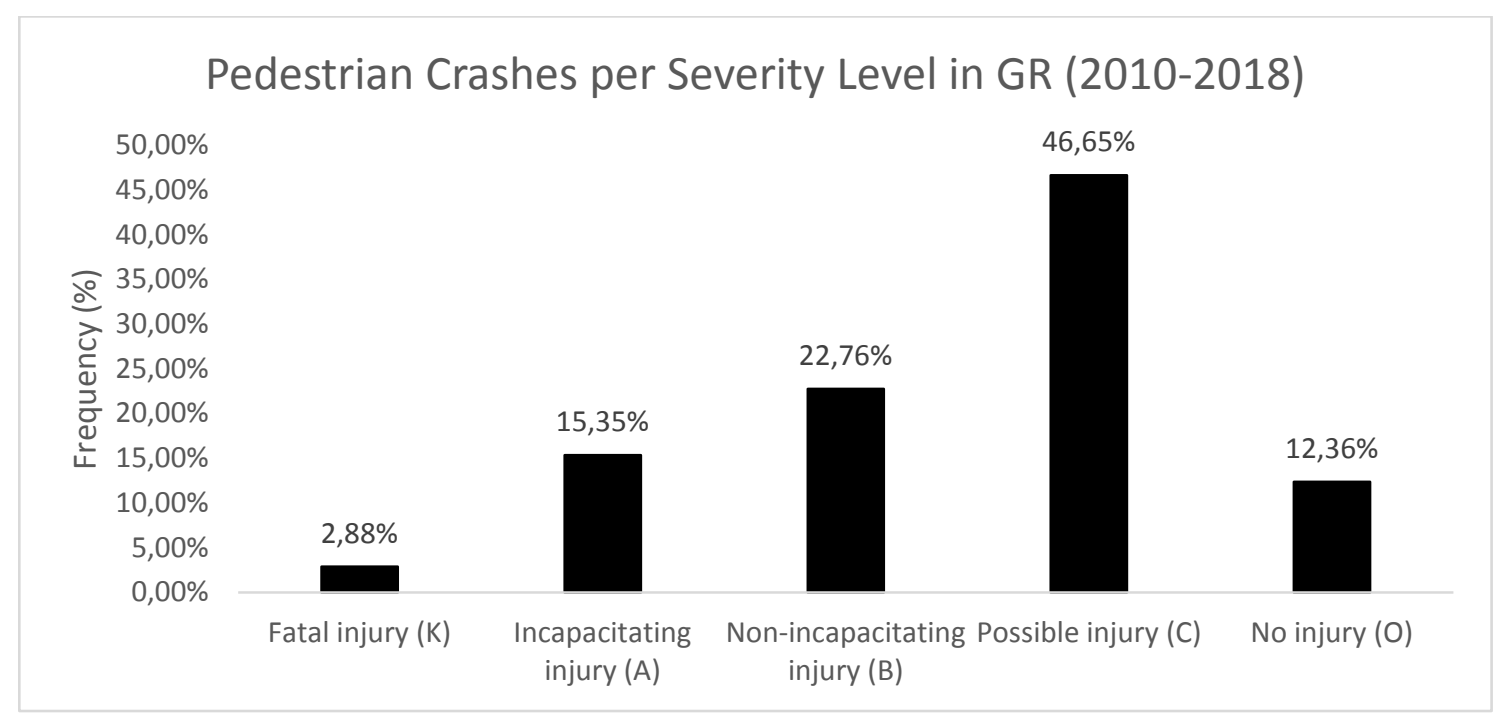

Figure 3. Pedestrian Crashes per Severity

\section{Crashes and Geometric Features of Selected Corridors and Intersections}

\subsection{Selected Crash Corridors}

There were five corridors identified from observed crash occurrence. Using a large print-out scale of Figure 2, we were able to identify crash cluster in the city of Grand Rapids. Crashes were observed based on the closeness to each other per type of severity: fatal, injury and non-injury, per year. Identified corridors were mostly in the downtown area of the city. Intersections within corridors were selected as target locations. From frequency, intersections are locations were most pedestrian crashes occur. A total of 113 crashes were observed at those intersections. Crashes at intersections were selected within a buffer of 250 feet as beyond that distance geometric characteristic changes. Crashes in these five corridors represent $10 \%$ of all crashes during the nine-year period.

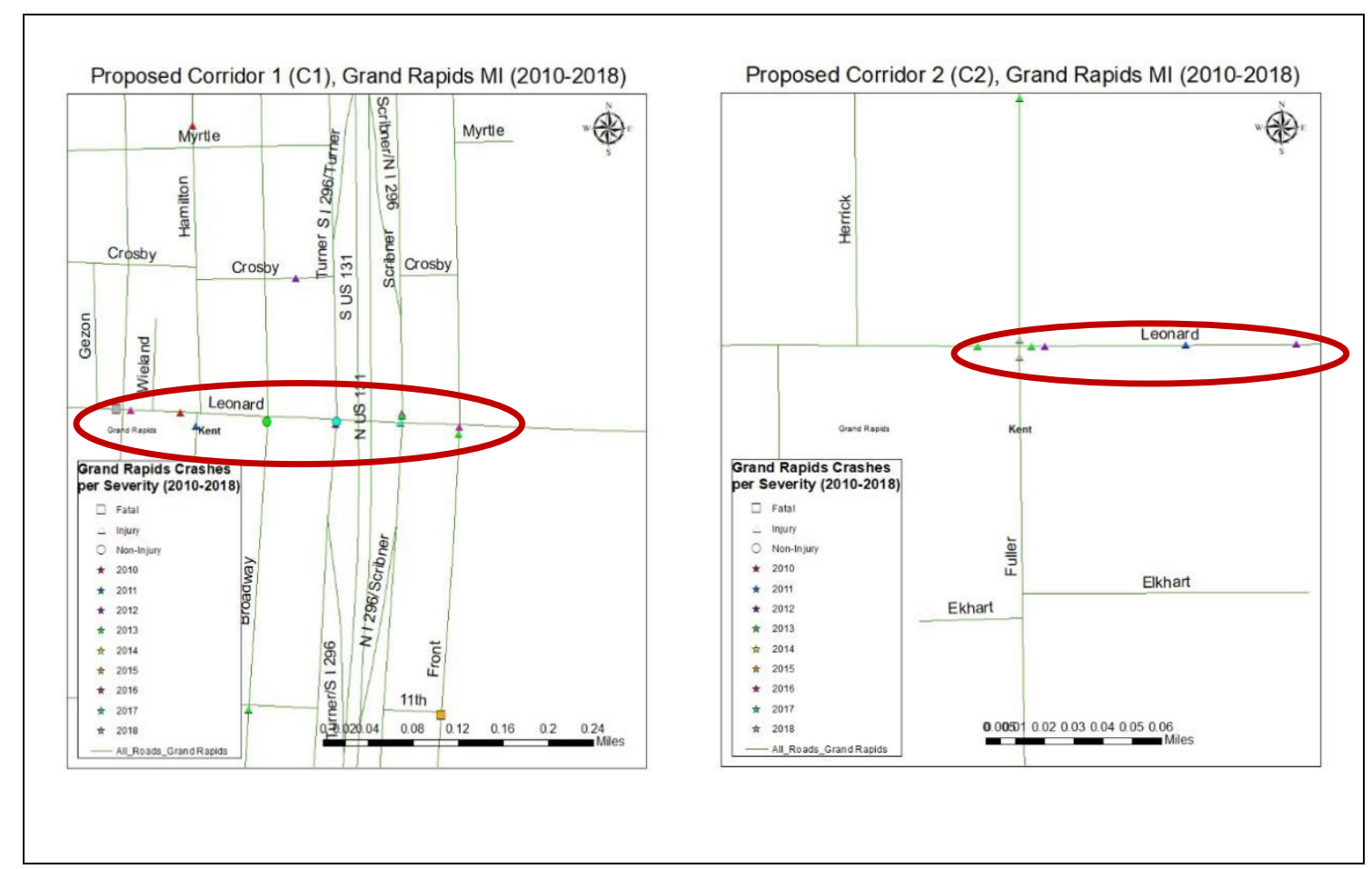

Figure 4a. Cluster of Crashes per Proposed Corridor in Grand Rapids 


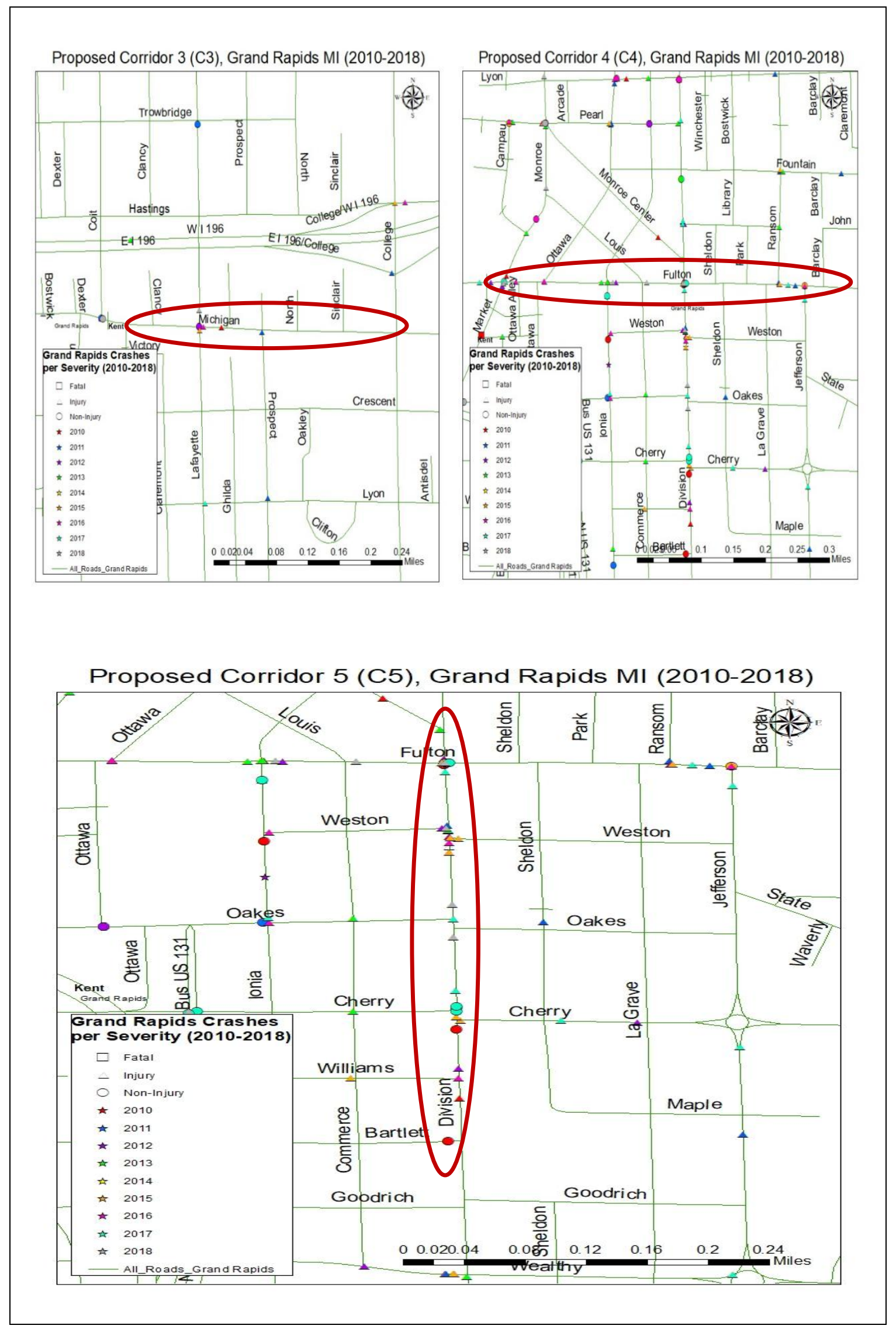

Figure 4b. Cluster of Crashes per Proposed Corridor in Grand Rapids 
Figure 4 (Composite figure of cluster of crashes) presents the corridors selected and the number of crashes observed. For corridor $1(\mathrm{C} 1)$, the main road is Leonard street and intersecting roads are Hamilton, Broadway, Turner, Scribner, and Front. Adding to a total of 13 crashes. In corridor 2 (C2), the main road is Leonard street as well, but now intersecting with Fuller. There were two mid-block crashes falling into the 250 feet buffer considered in this corridor NE from Fuller and Leonard street. Thus, the total number of crashes for corridor 2 were seven. Corridors 1 and 2 are almost one as the share the same main road: Leonard street. There is an interchange between the two corridors, which lead to dividing them. Corridor 3 (C3) is presented with main road being Michigan Avenue. The minor roads intersecting Michigan Ave are: Coit, Barclay, Lafayette, Prospect, and College. There were 16 crashes observed in this corridor.

There were 38 crashes observed in corridor 4 (C4) as presented in Figure 3. The main road was Fulton street. The intersecting road were Ionia, Division, Sheldon, Lagrave, Ransom, and Jefferson. For corridor 5 (C5) the main road was Division avenue with intersecting roads at Weston, Cherry, Williams, and Bartlett. There were 39crashes in total at this last corridor. Finally, the figures above show the pedestriancollision diagrams at 22 urban signalized and non-signalized intersections. In these diagrams, the locations of all pedestrian crashes were plotted using GIS. The figures also provide with additional information on the injury severity of the pedestrian using the following codes: square as fatal, triangle as injury and circle as non-injury. The year when the crash occurred was color-coded to differentiate it from the others.

\subsection{Geometric Features at Selected Intersections}

There were 22 intersections analysed in total with 113 crashes ranging from fatal to non-injury. Most of the intersections had Two Way Turning Left (TWLT) lanes (21 out of 22). The maximum and minimum number of lanes among intersections was 5 and 1, respectively. $72 \%$ of the intersections had a crosswalk. $72 \%$ of the intersections were signalized, $14 \%$ was STOP controlled and $14 \%$ did not have any intersection control at that time. Out of those that were signalized 9 intersection had Diagonal traffic signal arrangement while 7 had a Box-Span arrangement. More than half of the intersections had 4-LEGs (14 out of 22), but just 3 had pedestrian refuge islands. 18 intersections had pedestrian curbs as the American Disability Act (ADA) requests; and only 9 of the intersections had pushbutton call buttons. Out of all intersections, 16 had pedestrian countdown signals (PCS). Each intersection had a sidewalk, presence of road pavement markings, and light poles. All these features were obtained though using Google Earth images.

These features helped us to understand the condition surrounding the pedestrians at the crash location. For example, if there was a lack of crosswalks and/or clear pavement markings in one of the sites, that might have been a potential cause of the crash. The same reasoning could have been considered if the intersection were not controlled. However, this was not enough to understand the cause of the crashes. Thus, we decided to look at the human factors involved in the crash. In order to see a better a diagram of crashes and narrative of what occurred, we read the police UD-10 reports for each crash. These helped to identify crash patterns for each corridor. The following section covers this part of the analysis.

\subsection{Crash Patterns per Selected Corridors}

The crash patterns were identified by Looking at the UD-10 Police Reports for every crash within each corridor. This helped in identifying unsafe acts or behaviours from drivers to pedestrian and vice-versa. The police reports were also reviewed to identify factors that adversely affect the safety of pedestrian at the intersections. The crash location diagram and descriptions in the police reports of these 113 crashes were reviewed, and the crash patterns in general were as follows: left-turn conflicts, right-turn conflicts, unclear or lack of crosswalk or stop bar, and pedestrian under alcohol influence/or unsafe area.

\subsubsection{Left Turn Conflict}

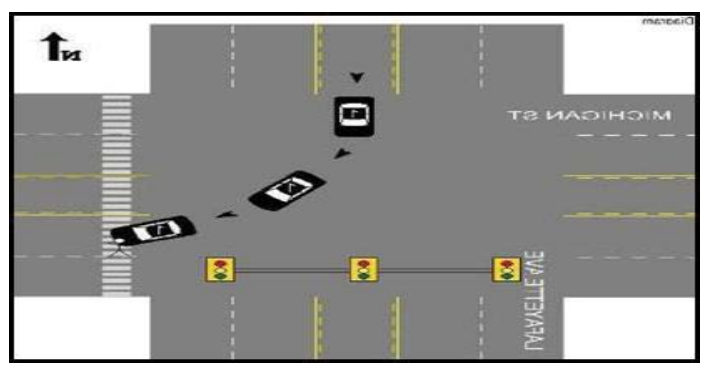

Figure 5. UD-10 of a left-turn Vehicle-Pedestrian Conflict

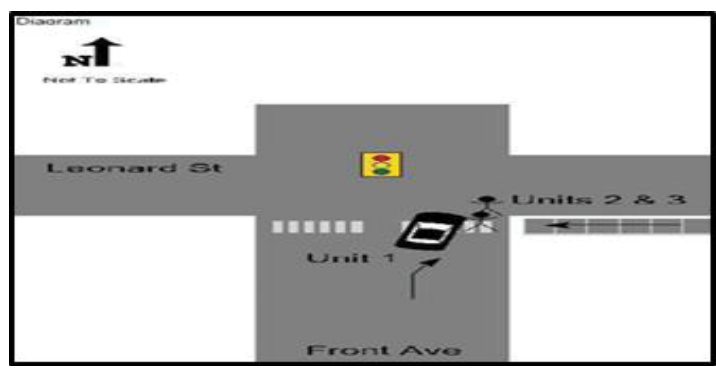

Figure 6. UD-10 of a right- turn Vehicle-Pedestrian Conflict 
Permissive left-turn phase at signalized intersection gives a turning left vehicle and the opposing through movement-traffic to proceed while the pedestrian signal is on: "WALK." Failure to yield to the pedestrian is a common pattern in this type of phase. A similar pattern was observed from crashes in Grand Rapids. For instance, Figure 5 shows a crash occurred at Michigan Ave with Lafyette Ave. In this case, the pedestrian was hit by a left-turning vehicle. Though a crosswalk was present, and the pedestrian reported to have the 'WALK' signal, the crash still happened. Intersections with permissive left turn phases have a higher number of vehicle-pedestrian conflicts. They can be eliminated by installing protected left-turn phases. They are recommended at intersections with high pedestrian activity. Also, when there is insufficient storage capacity for left-turning vehicles, wording would be added on signals next to the signals reminding drivers to yield right of way to pedestrians.

\subsubsection{Right-Turn Conflicts}

Right-turning vehicles must make a full stop when the traffic light is red. They are also supposed to yield to any pedestrian in their right to cross the intersection. However, not all drivers comply to this regulation and tend to fail to yield to pedestrians. For example, in the analysed corridors, many right-turns on red conflicts happened either by confusion or failure to yield. Figure 6 shows a crash involving two pedestrians and a right-turning vehicle at Leonard St and Front Ave. This type of crash could be eliminated by prohibiting right turn on red. In addition, at intersections suffering from a high rate of rightturn conflicts, a leading pedestrian (LP) interval might improve the pedestrian safety. LP provides the walk signal for extra 3-5 seconds before the concurrent through movement is on. Its makes pedestrians more visible before the motor vehicle. In this case the LPI will only help when there is a green light as drivers can turn right on red.

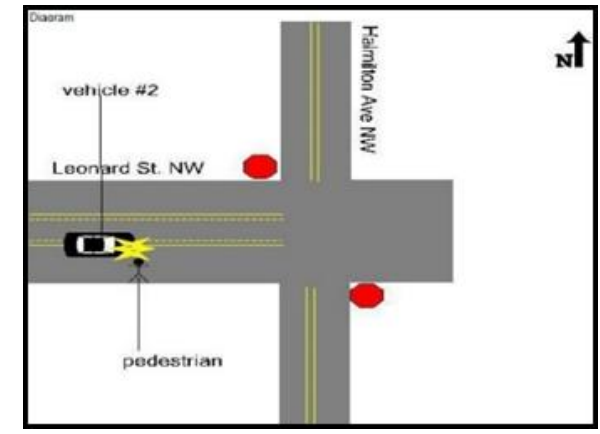

Figure 7. UD-10 of a Crash Occurring at intersection without Crosswalk

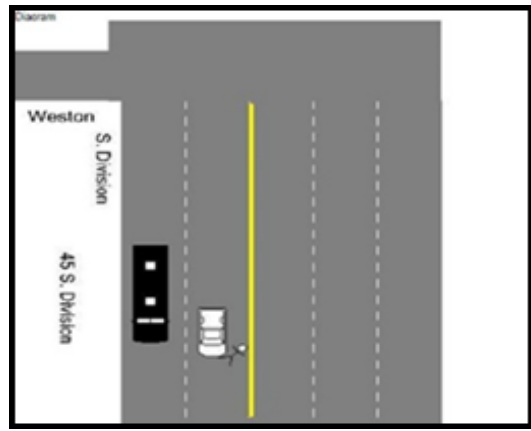

Figure 8. UD-10 of Drunk Pedestrian Crash

\subsubsection{Unclear/ Lack of Crosswalk or STOP Bar}

Many of the selected intersections did not have a crosswalk or in the case one was present; the markings were badly worn. For instance, Figure 7 shows one of the crashes occurring at Leonard St and Hamilton Ave clearly presents that situation. Besides the fact that the pedestrian was not crossing from the corner of the intersection, there was not crosswalk present as well. These types of crashes could have been prevented by painting a crosswalk or providing with an advance STOP bar.

\subsubsection{Pedestrian Under the Influence with Alcohol in Unsafe Area}

Another pattern that we observed was the one related to drunk pedestrians. This was mainly noticed at Division Avenue. Figure 8 shows the crash of a drunk pedestrian and a truck. The pedestrian entered the road recklessly and was hit. The behaviour could be due to a lack of pedestrian education and enforcement.

\section{Listed Crash Patterns and Potential Solutions per Corridors}

Several studies have been studied in order to support the potential solutions to the observed crash pattern in the city of Grand Rapids. The following paragraphs will provide a summary of them also it is shown in Table 2. 
Table 2. List of crash patterns and potential solutions

\begin{tabular}{|l|l|l|}
\hline Corridors & Crash Patterns & Potential solutions \\
\hline $1 \& 2$ & - Absence of crosswalk (8/12 crashes) & - Providing with an advance STOP bar 20 feet back \\
& - Unclear stop bar or crosswalk marking & - Pedestrian gateway R1-6 treatment at uncontrolled \\
& intersection (See Figure 8$)$
\end{tabular}

Van Houten et al., (2000) performed a field evaluation of a Leading Pedestrian (LP) signal phase. It was found that LPI signals significantly reduced conflicts between pedestrians and turning vehicles. It also reduced the incidences of pedestrians having to yield to vehicles. In 2017, Ivan et al., used LPI to reduce pedestrian vehicle conflicts at intersections. Pedestrians at concurrent phase signals are given a 'WALK' signal a few seconds prior to vehicles being given the green light. Zhang et al., (2015) made a comparison between exclusive and concurrent phasing intersections. The exclusive pedestrian phasing showed fewer crashes between pedestrians and motor vehicles than concurrent. Also, Van Houten et al., (2013) performed a study to observe the outcome from the presence of a high-visibility enforcement (pedestrian R1-6 gateway treatment) placed at mid-blocks. This treatment consistently produced marked increases in the percentage of drivers yielding to pedestrians.

Havard and Willis (2012) demonstrated that installing a marked crosswalk increased the givingright-of-way to pedestrians, and it also increased the usage of crosswalk. In addition, after the zebracrossing was installed, pedestrians felt safer, less vulnerable to traffic and more confident when crossing the road. In 1988, Van Houten found that providing with an advance STOP bar from crosswalk, resulted in an increase of vehicle compliance to pedestrian from $50 \%$ to $95 \%$. The percentage of drivers who yield to pedestrian increased from $32 \%$ (baseline) to $39 \%$.

\section{Conclusions and Recommendations}

This study analyzed vehicle-pedestrian crashes in the city of Grand Rapid Michigan from different perspectives. Firstly, the study identified the pedestrian population-crash ratio of largest cities in Michigan using 20,341 pedestrian crashes in Michigan from 2010-2018 and 2018 population from the United States Census Bureau. It was found that the city of Grand Rapid is the second largest city in Michigan with 1:198 ratio. Secondly, the study also identified the general trend of pedestrian and environmental characteristics that are correlated to pedestrian crashes using GIS. According to findings, fifty percent of the pedestrian crashes are possible injured, the most of crashes occurred at intersections, and $16 \%$ of pedestrians involved in crashes are intoxicated. Also, pedestrian crashes were observed to occur the most during lighted conditions: daylight and dark-lighted than unlighted condition.

Thirdly, the study identified corridors from observed crash occurrence using large print-out scale map. Intersections, buffer 250 feet, within corridors were selected as target locations. A total of 113 intersection pedestrian-crashes observed on five main corridors were investigated. It was found that most of intersection has two way turning left (TWLT), and the presence of crosswalk and traffic signals correlated to more crashes to absence of traffic signal. It was found that more than half of the intersection had 4-legs, and around $40 \%$ of these intersections had pushbutton call buttons. Finally, the study investigated the crash patterns using UD-10 police reports for every crash within each corridor. It appears that left-turn conflicts, right-turn conflicts, unclear or lack of crosswalk or stop bar, and pedestrian under alcohol influence were that most crash patterns. 
Finally, due to the study findings, several countermeasures of pedestrian crashes are suggested. For instance, providing with and advance STOP bar 20 feet back, place signage on 'prohibiting RTOR' when pedestrian present, providing with leading pedestrian interval phases, and providing pedestrian gateway R16 treatment at uncontrolled intersection. Based on the Crash patterns in the five selected corridors in the city of GR, it is concluded that the potential solutions presented could help in reduce the number of pedestrian crashes. Moreover, fields observations are recommended to better understand factors leading to these crashes. Furthermore, it is recommended to observe a land use map of the city of Grand Rapids. It was realized that one of the areas with a higher pedestrian volume was near a hospital in Michigan Avenue. Conclusively, the city of Grand Rapids is working towards reducing then number of crashes, including pedestrians, every year. Currently, the intersection of Leonard and Hamilton is being reconstructed with crosswalks (which are not present in the previous years). Also, 8-mile of bike lanes has been painted around the Grand Rapids, making the city more liveable. We are hopeful on the recommended solutions as we expect them to contribute to the goal of the city and the state department of transportation (MDOT): towards zero death goals.

\section{Acknowledgement}

The authors would like to acknowledge Dr. Van Houten for his academic support, encouragement and enthusiasm in the study and its developments. Also, the authors acknowledge Christopher Zull for his time and support in explaining the improvements/actions taken in the city of Grand Rapids.

\section{References}

1. Alhajyaseen, W. K. M., Asano, M., and Nakamura, H. (2013) Left-turn gap acceptance models considering pedestrian movement characteristics. Accident Analysis and Prevention.

2. Ang, B. H., Chen, W. S., and Lee, S. W. H. (2017) Global burden of road traffic accidents in older adults: A systematic review and meta-regression analysis. Archives of Gerontology and Geriatrics.

3. Chen, F., Peng, H., Ma, X., Liang, J., Hao, W., and Pan, X. (2019) Examining the safety of trucks under crosswind at bridge-tunnel section: A driving simulator study. Tunnelling and Underground Space Technology.

4. Du, R., Qiu, G., Gao, K., Hu, L., and Liu, L. (2020) Abnormal road surface recognition based on smartphone acceleration sensor. Sensors (Switzerland).

5. Havard, C., and Willis, A. (2012) Effects of installing a marked crosswalk on road crossing behaviour and perceptions of the environment. Transportation Research Part F: Traffic Psychology and Behaviour.

6. Van Houten, R. (1988) The effects of advance stop lines and sign prompts on pedestrian safety in a crosswalk on a multilane highway. Journal of Applied Behavior Analysis.

7. Van Houten, R., Malenfant, L., Huitema, B., and Blomberg, R. (2013) Effects of high-visibility enforcement on driver compliance with pedestrian yield right-of-way laws. Transportation Research Record.

8. Van Houten, R., Retting, R. A., Farmer, C. M., and Van Houten, J. (2000) Field evaluation of a leading pedestrian interval signal phase at three urban intersections. Transportation Research Record.

9. Iryo-Asano, M., Alhajyaseen, W. K. M., and Nakamura, H. (2015) Analysis and Modeling of Pedestrian Crossing Behavior during the Pedestrian Flashing Green Interval. IEEE Transactions on Intelligent Transportation Systems.

10. Ivan, J. N., McKernan, K., Zhang, Y., Ravishanker, N., and Mamun, S. A. (2017) A study of pedestrian compliance with traffic signals for exclusive and concurrent phasing. Accident Analysis and Prevention.

11. Kim, D. (2019) The transportation safety of elderly pedestrians: Modeling contributing factors to elderly pedestrian collisions. Accident Analysis and Prevention.

12. Kim, J. K., Ulfarssom, G. F., Shankar, V. N., and Mannering, F. L. (2010) A note on modeling pedestrian-injury severity in motor-vehicle crashes with the mixed logit model. Accident Analysis and Prevention.

13. Lam, W. W. Y., Yao, S., and Loo, B. P. Y. (2014) Pedestrian exposure measures: A time-space framework. Travel Behaviour and Society.

14. Marshall, W. E., and Garrick, N. W. (2011) Does street network design affect traffic safety? Accident Analysis and Prevention. 
15. Newnam, S., Blower, D., Molnar, L., Eby, D., and Koppel, S. (2018) Exploring crash characteristics and injury outcomes among older truck drivers: An analysis of truck-involved crash data in the United States. Safety Science.

16. O’Donovan, S., van den Huevel, C., Baldock, M., and Byard, R. W. (2018) Factors involved in the assessment of paediatric traffic injuries and deaths. Medicine, Science and the Law.

17. Prato, C. G., Kaplan, S., Patrier, A., and Rasmussen, T. K. (2019) Integrating police reports with geographic information system resources for uncovering patterns of pedestrian crashes in Denmark. Journal of Transport Geography.

18. Rankavat, S., and Tiwari, G. (2016) Pedestrians risk perception of traffic crash and built environment features - Delhi, India. Safety Science.

19. Tulu, G. S., Washington, S., Haque, M. M., and King, M. J. (2015) Investigation of pedestrian crashes on two-way two-lane rural roads in Ethiopia. Accident Analysis and Prevention.

20. Wang, X., Yang, J., Lee, C., Ji, Z., and You, S. (2016) Macro-level safety analysis of pedestrian crashes in Shanghai, China. Accident Analysis and Prevention.

21. Wang, Y., and Kockelman, K. M. (2013) A Poisson-lognormal conditional-autoregressive model for multivariate spatial analysis of pedestrian crash counts across neighborhoods. Accident Analysis and Prevention.

22. Yao, S., Loo, B. P. Y., and Lam, W. W. Y. (2015) Measures of activity-based pedestrian exposure to the risk of vehicle-pedestrian collisions: Space-time path vs. potential path tree methods. Accident Analysis and Prevention.

23. Zhang, Y., Mamun, S. A., Ivan, J. N., Ravishanker, N., and Haque, K. (2015) Safety effects of exclusive and concurrent signal phasing for pedestrian crossing. Accident Analysis and Prevention. 